\title{
First-principles study of field emission from carbon nanotubes and graphene nanoribbons
}

\author{
Joseph A. Driscoll, Brandon Cook, Sergiy Bubin, and Kálmán Varga \\ Department of Physics and Astronomy, Vanderbilt University, Nashville, Tennessee 37235, USA
}

(Received 17 April 2011; accepted 11 June 2011; published online 21 July 2011)

\begin{abstract}
A real-space, real-time implementation of time-dependent density functional theory is used to study electron field emission from nanostructures. Carbon nanotubes and graphene nanoribbons are used as model systems. The calculations show that carbon nanotubes with iron adsorbates have spin-polarized emission currents. Graphene nanoribbons are shown to be good field emitters with spatial variation of the emission current influenced by the presence of passivating hydrogen. () 2011 American Institute of Physics. [doi:10.1063/1.3610511]
\end{abstract}

\section{INTRODUCTION}

Field emission (FE) from nanostructures is the subject of intense experimental and theoretical research. Electron field emitters may be used in next-generation displays, electron sources, ${ }^{1-4}$ and high-resolution electron beam instruments. ${ }^{5-8}$ In order for electrons to escape a material and be emitted, they must somehow move beyond the material's confining potential barrier. Electrons can be given enough energy to escape by heating the system in a process called thermionic electron emission. ${ }^{9}$ Alternatively the barrier can be lowered by the application of an external electric field, allowing electrons to tunnel out. The latter process is known as electron field emission. ${ }^{1}$ Schottky emitters ${ }^{10,11}$ combine thermionic emission with electric field-based lowering of the material's work function. This lowering, known as the Schottky effect, ${ }^{12}$ makes it easier for thermally excited electrons to escape the material.

Disadvantages of thermionic emission include large power consumption and potential heat damage to the device. ${ }^{13}$ While field emitters do not directly use heating to emit electrons, materials used in field emission can become damaged due to current-induced heating. Using a device such as a carbon nanotube helps, ${ }^{13}$ since it is more robust to such effects. In addition, field emitters can respond to varying electric fields much faster than a thermionic emitter can alter its output, meaning that field emitters are capable of operation at higher frequencies. ${ }^{14}$

Compared with thermionic emission, nanotube field emitters produce a narrow beam size. ${ }^{7}$ This is important for high-resolution displays and spatial microscopy. Another useful property of the electrons emitted from a nanotube is their narrow energy spectrum, ${ }^{15}$ important in, e.g., energyresolved microscopy.

Nanoscale electron field emitters have been extensively studied both theoretically and experimentally. ${ }^{1-8}$ The aim of these studies is to explore the properties of nanoscale materials in electric fields and exploit these properties for technological applications. The standard approach to modeling field emission is Fowler-Nordheim theory, ${ }^{16}$ which describes electron emission from a flat metal surface in the presence of an electric field. With nanostructures, some aspects of the experimental data can be modeled in this way. ${ }^{17}$ But in other cases, for example with high fields, the theory fails to describe the phenomena. ${ }^{18}$

Beyond Fowler-Nordheim theory, there are more rigorous methods that take into account atomic geometry and electronic structure. ${ }^{19-22}$ These include first-principles calculations, ${ }^{23,24}$ in which the self-consistent electronic structure of the field emitter is obtained and the wave function is matched to the asymptotic scattering wave function of the electrons in the external field. ${ }^{20,21}$ An important step beyond these static calculations is the introduction of the timedependent description of FE. ${ }^{23,24}$

In the time-dependent approach the wave function is time-propagated to describe the effect of the electric field. This approach has several advantages. In the time-independent approach, the wave function has to be matched to the asymptotic wave function. At the same time, the asymptotic wave function, the wave function of electrons in an electric field, is not known. It is usually approximated by Airy functions, which are the wave functions of independent noninteracting electrons in an electric field. This approximation is avoided in the time-dependent approach: The field-emitted electrons and the electrons of the emitter are described on an equal footing. The asymptotic form of the wave function is not needed in the calculation, and the time evolution of the wave function is used to describe the emitted current.

So far, time-dependent approaches have usually been limited to time propagation of the wave functions with a time-independent ground state Hamiltonian. The calculations presented here go beyond this and use a time-dependent Hamiltonian. Our approach simulates the entire field emission process in a real-time, real-space framework based on time-dependent density functional theory (TDDFT). Previously ${ }^{25}$ the method has been used to study the effects of adsorbates on nanotube field emission. In this paper we predict spin-polarized field emission from carbon nanotubes. We also investigate the impact of passivating hydrogen on the field emission from graphene nanoribbons. Following this Introduction, Sec. II discusses the theoretical framework for our calculations, and Sec. III presents our results. A summary is given in Sec. IV. 


\section{FORMALISM}

The calculations presented here have been performed using a Lagrange function ${ }^{26}$ based real-space, real-time implementation of TDDFT. ${ }^{27}$ Electron dynamics in TDDFT are described by the time-dependent Kohn-Sham equation

$$
i \hbar \frac{\partial}{\partial t} \Psi_{k}^{\sigma}(\mathbf{r}, t)=H^{\sigma} \Psi_{k}^{\sigma}(\mathbf{r}, t)
$$

where $\sigma$ indicates the spin state $(\sigma \in\{\uparrow, \downarrow\})$ and $\Psi_{k}^{\sigma}$ is the $k^{\text {th }}$ Kohn-Sham orbital in spin state $\sigma$. The spin-dependent Kohn-Sham Hamiltonian is

$$
\begin{aligned}
H^{\sigma}= & -\frac{\hbar^{2}}{2 m} \nabla_{\mathbf{r}}^{2}+V_{A}(\mathbf{r}, t)+V_{H}[n](\mathbf{r}, t) \\
& +V_{X C}^{\sigma}\left[n_{\uparrow}, n_{\downarrow}\right](\mathbf{r}, t)+V_{\text {ext }}[n](\mathbf{r}, t) .
\end{aligned}
$$

$V_{\text {ext }}(\mathbf{r}, t)$ is a time-dependent external potential (e.g., an applied electric field). $V_{A}(\mathbf{r}, t)$ is the atomic potential, which we represent with pseudopotentials. ${ }^{28}$ The Hartree potential $V_{H}(\mathbf{r}, t)$ is found by solving the Poisson equation for the total electron density $n(\mathbf{r}, t)$, which is calculated from the KohnSham orbitals $\Psi_{k}^{\sigma}$ as

$$
n(\mathbf{r}, t)=\sum_{\sigma} n_{\sigma}(\mathbf{r}, t), \quad n_{\sigma}(\mathbf{r}, t)=\sum_{k}\left|\Psi_{k}^{\sigma}(\mathbf{r}, t)\right|^{2}
$$

Exchange and correlation effects are included in $V_{X C}^{\sigma}(\mathbf{r}, t)$. In spin-dependent calculations the local spin density approximation (LSDA) ${ }^{29}$ is used, otherwise we use the local density approximation (LDA) with the parameterization of Perdew and Zunger. ${ }^{30}$

The orbitals, $\Psi_{k}^{\sigma}(\mathbf{r}, t)$, are represented on uniform realspace grids. A spacing of $0.33 \AA$ in all three spatial dimensions is found to provide good accuracy and is used in all calculations. Compared to localized bases such as atomic orbitals the grid representation is particularly convenient for describing current-carrying states because it can describe the time-dependent electron density accurately not only near atomic centers, but throughout the entire simulation volume.

To solve the time-dependent Kohn-Sham equation [Eq. (1)] we time propagated the Kohn-Sham orbitals using a Taylor expansion of the exponential time propagator: ${ }^{31}$

$$
\Psi_{k}^{\sigma}(\mathbf{r}, t+\Delta t)=\sum_{j=0}^{N_{T}} \frac{(i \hbar)^{j}}{j^{!}}\left(H^{\sigma}\right)^{j} \Psi_{k}^{\sigma}(\mathbf{r}, t),
$$

where $\Delta t$ is the time step (we used $0.001 \mathrm{fs}$ ) and the order of the Taylor expansion is taken to be $N_{T}=4$. The system's ground state was used as the initial state $\Psi_{k}^{\sigma}(\mathbf{r}, t=0)$. During the time development we calculated the field emission current density at a time $t$ using

$$
j^{\sigma}(\mathbf{r}, t)=\frac{e \hbar}{2 i m} \sum_{k}\left(\left[\Psi_{k}^{\sigma}\right]^{*} \nabla_{\mathbf{r}} \Psi_{k}^{\sigma}-\Psi_{k}^{\sigma} \nabla_{\mathbf{r}}\left[\Psi_{k}^{\sigma}\right]^{*}\right) .
$$

In a time-dependent calculation, it is possible that the electron density can reach the boundary of the finite simulation volume and produce nonphysical reflections. The reflections can be prevented by the addition of a complex absorbing potential (CAP) which absorbs electron density near the boundaries. The CAP is zero in the region of the nanostructure and where the current is measured, and so does not impact the results. Various types of complex absorbing potentials have been developed; ${ }^{32,33}$ we use the form developed by Manolopoulos ${ }^{34}$ with a small modification. Formally, the strength of this CAP approaches infinity at the boundary; this may introduce numerical instabilities in calculations with numerical grids. Such instabilities can be eliminated by slightly shifting the position of the CAP so that its singularity occurs just outside of the simulation volume. Note that the CAP does not include any time or spin dependence. The CAP is introduced into the simulation by adding it to the Hamiltonian in Eq. (2).

\section{RESULTS}

In this section we present calculations of field emission from carbon nanotubes and graphene nanoribbons. Carbon nanotubes with and without Fe adsorbates and graphene nanoribbons with and without passivating hydrogens are studied. We also discuss some details of the computations and consider the effect of ionic motion.

After the ground state of the system is calculated the electric field is added and time development begins. The electric field is directed along the long axis of the nanostructure. The electric field's magnitude is increased up to its maximum value with a linear ramp over $0.2 \mathrm{fs}$. The ramping is used to avoid an instantaneous appearance of the field and introduction of an abrupt jump. Such jumps are unphysical, as an instantaneous jump is not possible in real experiments. At time $t$, the field strength $E$ is given by

$$
E(t)=E_{M} \min \left(1, \frac{t}{t_{\mathrm{ramp}}}\right)
$$

where $E_{M}$ is the maximum electric field magnitude and $t_{\mathrm{ramp}}$ is the duration of the ramping. The ramping duration of 0.2 fs was determined empirically to be sufficient to avoid transient effects. FE currents are determined once a steady state is reached.

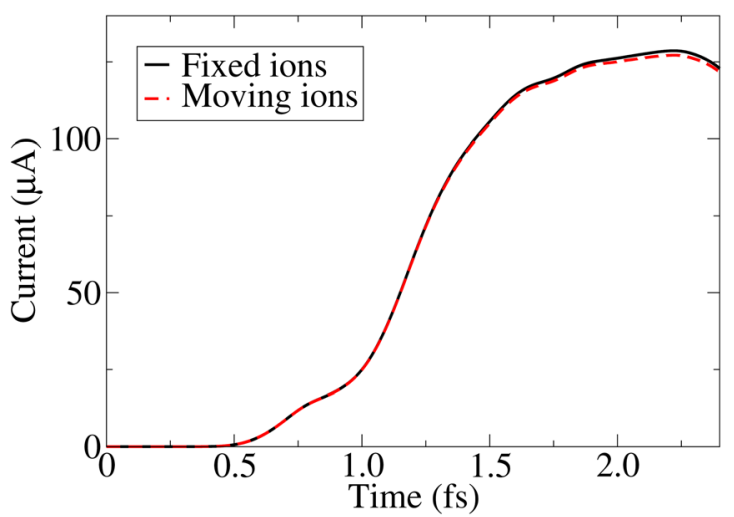

FIG. 1. (Color online) Field emission current vs time for a nanotube with and without moving ions. 


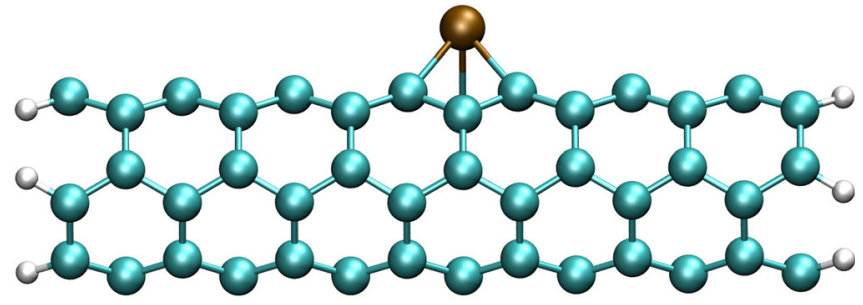

FIG. 2. (Color online) Structure of a $(3,3)$ carbon nanotube with an Fe atom adsorbed on the side.

\section{A. Ionic motion}

Our previous atomistic simulations of field emission have assumed that the position of nuclei and core electrons are fixed. To test this fixed ion approximation we will compare field emission from a $(3,3)$ carbon nanotube with and without moving ions. Ions are moved classically under the influence of quantum forces (i.e., Ehrenfest molecular dynamics ${ }^{35}$ ). The motion of ions is described by:

$$
\begin{aligned}
M_{i} \frac{d^{2} \mathbf{R}_{i}}{d t^{2}}= & -\nabla_{\mathbf{R}_{i}}\left[Z_{i} V_{\text {ext }}\left(\mathbf{R}_{i}, t\right)+\sum_{i<j}^{N_{\text {ion }}} \frac{Z_{i} Z_{j}}{\left|\mathbf{R}_{i}-\mathbf{R}_{j}\right|}\right. \\
& \left.+\int V_{A}\left(\mathbf{r}, \mathbf{R}_{i}\right) n(\mathbf{r}, t) d \mathbf{r}\right],
\end{aligned}
$$

where $M_{i}, Z_{i}$, and $\mathbf{R}_{i}$ are the mass, pseudocharge (valence), and position of the $i^{\text {th }}$ ion, respectively. The ionic motion is coupled with the Kohn-Sham equations through the density, $n(\mathbf{r}, t)$. At each time step the positions and velocities of ions are updated with the Verlet algorithm. ${ }^{36}$

Figure 1 shows the field emission current versus time for a nanotube with and without moving ions. The curves are nearly identical, establishing the reliability of the fixed-ion approximation for these calculations. In this case the ions moved very little, approximately $0.001 \AA$ per ion. We note that ionic motion could become important for longer simulations.

\section{B. Spin-polarized field emission from nanotubes}

Spin-polarized field emitters are useful in, for example, spin-resolved electron microscopy, ${ }^{37}$ which allows study of the magnetic structure of materials. Spin-polarized field emission has been demonstrated with EuS-coated tungsten tips, ${ }^{38,39}$ GaAs emitters, ${ }^{40}$ and other systems. Hoa et al.

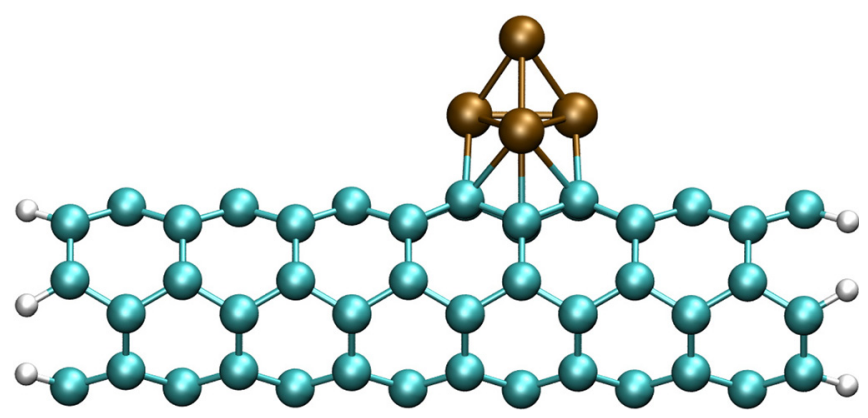

FIG. 3. (Color online) Structure of a (3,3) carbon nanotube with an $\mathrm{Fe}_{4}$ cluster adsorbed on the side.
TABLE I. Peak spin-polarized field emission currents for $(3,3)$ nanotubes with different adsorbates in a $1 \mathrm{~V} / \AA ̊$ electric field. Currents are in $\mu \mathrm{A}$.

\begin{tabular}{lcccc}
\hline \hline Adsorbate & $I_{\text {up }}$ & $I_{\text {down }}$ & $I_{\text {total }}$ & Polarization \\
\hline None & 71.6 & 71.6 & 143 & $0 \%$ \\
$\mathrm{Fe}$ & 55.7 & 68.2 & 124 & $10.1 \%$ \\
$\mathrm{Fe}_{4}$ & 48.8 & 63.9 & 113 & $13.4 \%$ \\
\hline \hline
\end{tabular}

calculated the electronic structure of Mn-doped GaN nanotubes and predict spin-polarized field emission. ${ }^{41,42}$ In contrast, we study carbon nanotubes, due to their greater industrial availability and other favorable properties relative to GaN nanotubes. Zhang et $a l .{ }^{43}$ have shown that various metal atoms, including iron, readily form clusters on the surface of carbon nanotubes. However, spin-polarized field emission from carbon nanotubes with iron adsorbates has not yet been experimentally demonstrated. In this section spindependent field emission calculations for carbon nanotubes are presented. The effect of various adsorbates (Fe atoms or clusters) is examined.

We study a $(3,3)$ carbon nanotube with and without iron adsorbates. Figures 2 and 3 show the nanotube with an adsorbed $\mathrm{Fe}$ atom and $\mathrm{Fe}_{4}$ cluster, respectively. Coordinates for the cluster were obtained from Yuan et al. ${ }^{44}$ Dangling bonds at the ends of the nanotube are passivated with hydrogen atoms. The field emission from a nanotube with no adsorbates was calculated to establish a baseline for evaluating spin-polarized currents from nanotubes with adsorbates. Optimized geometries are obtained via spin-polarized DFT calculations using the $\operatorname{VASP}^{45}$ ab initio package.

Table I summarizes the peak spin-polarized currents for the systems tested. The spin up, spin down, and total currents are indicated in the table by $I_{\mathrm{up}}, I_{\mathrm{down}}$, and $I_{\text {total }}$, respectively.

The table also provides the degree of spin polarization in each case, defined as

$$
\text { Polarization }=\frac{\left|I_{\text {up }}-I_{\text {down }}\right|}{I_{\text {total }}} \times 100 \% .
$$

The applied field had a magnitude of $1.0 \mathrm{~V} / \AA$ and was directed along the axis of the nanotube. For the bare (no adsorbate) nanotube, spin polarization was not present; the

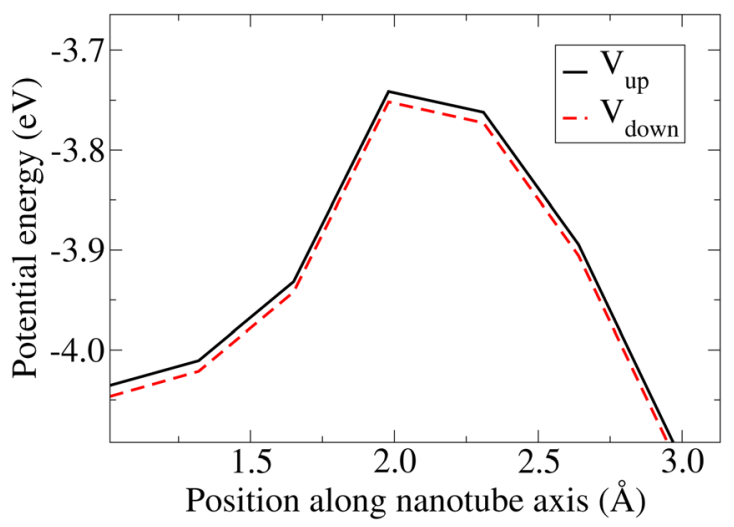

FIG. 4. (Color online) Ground state potential energy projections for a nanotube with an adsorbed $\mathrm{Fe}_{4}$ cluster. The projection is made onto the axis along the nanotube. 


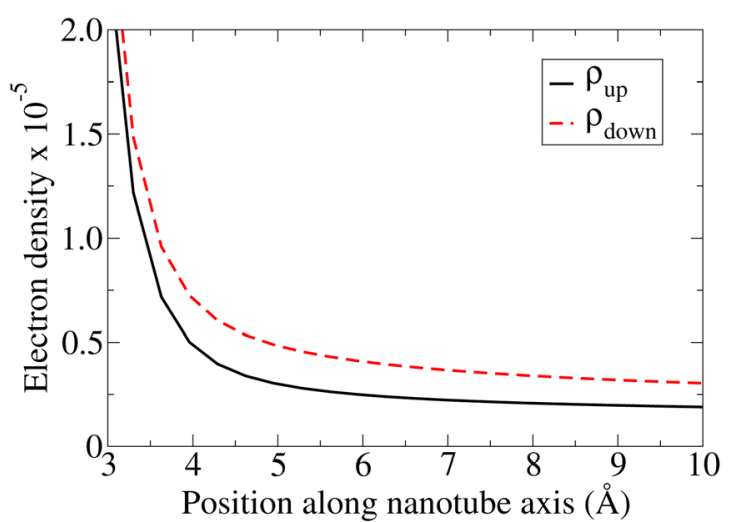

FIG. 5. (Color online) Ground state density projections for a nanotube with an adsorbed $\mathrm{Fe}_{4}$ cluster.

numbers of spin up and spin down electrons were the same. So, as expected, the values for the spin up and spin down currents were identical and equal to one-half of the total current. The spin up and spin down currents for the nanotubes with iron adsorbates are significantly different, with the spin down current exceeding the spin up current. Addition of either of the iron adsorbates reduces the total current of the nanotube as compared to the no adsorbate case. The reduction is more pronounced for the case of the $\mathrm{Fe}_{4}$ cluster, which suggests that the reduction is due to scattering effects. For both up and down spin currents, the Fe atom adsorbate produces a higher current than the $\mathrm{Fe}_{4}$ cluster. The degree of polarization is relatively low, but these calculations were done for single adsorbates; future work is needed to determine the polarization when using more adsorbates. The presence of both a single $\mathrm{Fe}$ atom and an $\mathrm{Fe}_{4}$ cluster are shown to cause separations in the spin up and spin down currents, indicating that carbon nanotubes with iron adsorbates can be used as spin-polarized current sources.

The mechanism of spin-polarization of the current with adsorbates can be elucidated by potential energy and density profiles for the nanotube with an adsorbed $\mathrm{Fe}_{4}$ cluster. Figure 4 shows that the tunneling barrier is reduced for down spin electrons, which leads to more current for that spin state. Figure 5 shows that at the ground state, the down electronic density was already more spread out from the nanotube.

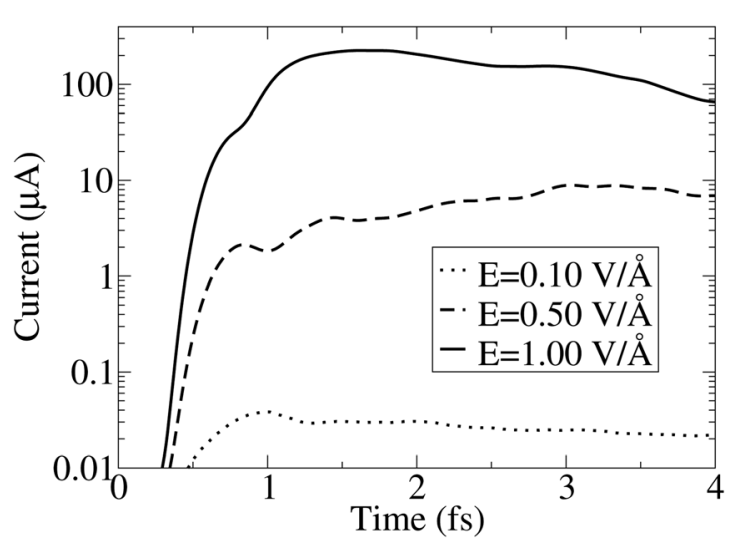

FIG. 6. Field emission current vs time for a $(3,3)$ graphene nanoribbon. Curves for three different applied electric fields are shown.
TABLE II. Peak field emission currents for $(3,3)$ carbon nanotubes $(\mathrm{CNT})$ and graphene nanoribbons (GNR) in varying electric fields. " $+\mathrm{H}$ " indicates that passivating hydrogens were present on the emission edge. Currents are in $\mu \mathrm{A}$.

\begin{tabular}{lccc}
\hline \hline & $0.10 \mathrm{~V} / \AA$ & $0.50 \mathrm{~V} / \AA$ & $1.00 \mathrm{~V} / \AA$ \\
\hline $\mathrm{CNT}$ & 0.0321 & 30.6 & 266 \\
$\mathrm{CNT}+\mathrm{H}$ & 0.0351 & 51.4 & 279 \\
$\mathrm{GNR}$ & 0.0384 & 8.90 & 226 \\
$\mathrm{GNR}+\mathrm{H}$ & 0.0626 & 2.63 & 218 \\
\hline
\end{tabular}

\section{Field emission from graphene nanoribbons}

In this section we consider the field emission behavior of graphene nanoribbons (GNRs). Hydrogen passivation is used to satisfy the dangling bonds on the edges of the ribbons. The field emission behavior with and without hydrogens on the edge that emits is examined. In all cases, the nonemitting edges are passivated by hydrogens.

Figure 6 shows the field emission current versus time of a $(3,3)$ GNR without hydrogen passivation for different electric fields. Table II gives the remainder of the results. The calculations show the presence of the hydrogens reduces the current for GNRs. The effect of hydrogen passivation of the emitting edge on the spatial distribution of charge density is shown in Fig. 7. When there are no passivating hydrogens at the emission edge the current is higher and the emitted density has a more pronounced vertical splitting.

One can imagine rolling these ribbons up (along the emission axis) into nanotubes. The field emission performance of these corresponding nanotubes was also calculated to allow comparison to the nanoribbons. Figure 8 shows that a lack of hydrogens causes splits in the emitted density in the CNT case as well.

Field emission current for the GNRs increases by several orders of magnitude with increasing applied electric field (note the logarithmic scale in Fig. 6). The response is greater than in the case of nanotubes ${ }^{25}$ and the time required to reach a stable current is short. However, CNTs are seen to produce higher currents than corresponding GNRs. Addition of hydrogens to the emission edge of the nanostructure increases the current for the CNT, but decreases it for the GNR case. This could reflect differences between CNTs and GNRs in the local density of states in the emitting edge region.

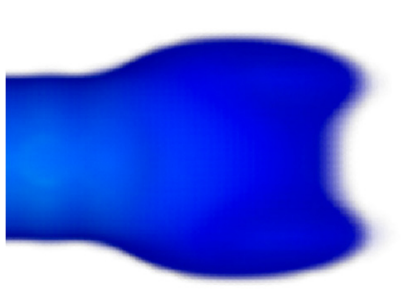

(a) With hydrogens

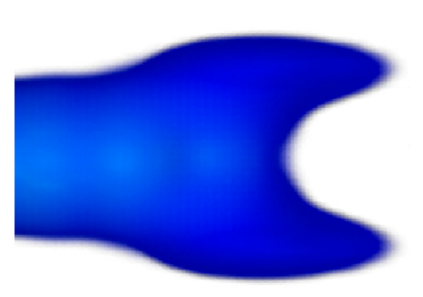

(b) Without hydrogens
FIG. 7. (Color online) Side view of electron density of GNR during peak emission (a) with and (b) without hydrogens at the emission edge. The emission edge is at the right of the figure. 


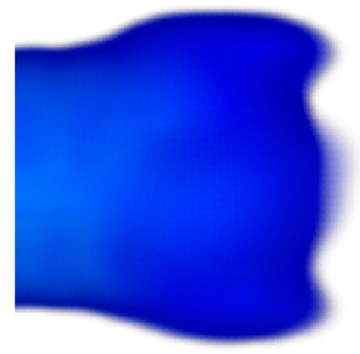

(a) With hydrogens

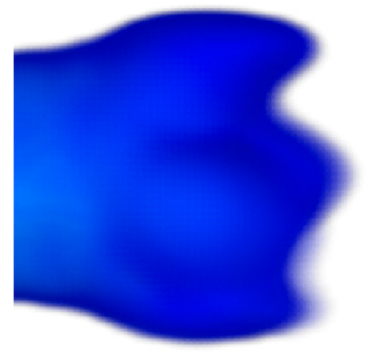

(b) Without hydrogens
FIG. 8. (Color online) Side view of electron density of CNT during peak emission (a) with and (b) without hydrogens at the emission edge. The emission edge is at the right of the figure.

\section{SUMMARY}

We have described our first-principles framework based on time-dependent density functional theory for studying electron field emission from nanostructures. Using this framework we have studied field emission from graphene nanoribbons and carbon nanotubes. Field emission currents with various applied static electric fields have been computed. In the case of nanotubes, the presence of iron adsorbates is shown to yield spin-polarized field emission.

Calculations show that high currents with relatively fast onset of steady states are possible with graphene nanoribbons. In addition, removal of passivating hydrogens from GNRs produces a pronounced variation in the spatial density distribution.

Due to the high computational cost of our first-principles calculations, there are many system variations that we were unable to include in the present work. Future calculations will include the use of other adsorbates to determine their impact on spin-polarized field emission. Along with Fe, metals such as Au and Al have also been shown ${ }^{43}$ to form stable clusters on the sides of nanotubes. It would also be interesting to study the spin polarization effects of multiple clusters on a single nanotube.

\section{ACKNOWLEDGMENTS}

This work has been supported by NSF grant CMMI0927345.

${ }^{1}$ W. de Heer, A. Châtelain, and D. Ugarte, Science 270, 1179 (1995).

${ }^{2}$ Y. Saito, K. Hamaguchi, K. Hata, K. Uchida, Y. Tasaka, F. Ikazaki, M. Yumura, A. Kasuya, and Y. Nishina, Nature 389, 554 (1997).

${ }^{3}$ W. Choi, D. Chung, J. Kang, H. Kim, Y. Jin, I. Han, Y. Lee, J. Jung, N. Lee, and G. Park, Appl. Phys. Lett. 75, 3129 (1999).
${ }^{4}$ A. Rinzler, J. Hafner, P. Nikolaev, P. Nordlander, D. Colbert, R. Smalley, L. Lou, S. Kim, and D. Tomanek, Science 269, 1550 (1995).

${ }^{5}$ Y. Saito and S. Uemura, Carbon 38, 169 (2000).

${ }^{6}$ K. A. Dean and B. R. Chalamala, J. Appl. Phys. 85, 3832 (1999).

${ }^{7}$ N. de Jonge, Y. Lamy, K. Schoots, and T. Oosterkamp, Nature 420, 393 (2002).

${ }^{8}$ H. Schmid and H.-W. Fink, Appl. Phys. Lett. 70, 2679 (1997).

${ }^{9}$ O. Richardson, Science 38, 57 (1913).

${ }^{10}$ H. Kim, M. Yu, M. Thomson, E. Kratschmer, and T. Chang, J. Appl. Phys. 81, 461 (1997).

${ }^{11}$ L. W. Swanson, J. Vac. Sci. Technol. 12, 1228 (1975).

${ }^{12}$ C. H. Garcia and C. A. Brau, Nucl. Instrum. Methods Phys. Res. A 483, 273 (2002).

${ }^{13}$ W. Milne, K. Teo, G. Amaratunga, P. Legagneux, L. Gangloff, J. Schnell, V. Semet, V. Binh, and O. Groening, J. Mater. Chem. 14, 933 (2004).

${ }^{14}$ K. Teo, E. Minoux, L. Hudanski, F. Peauger, J. Schnell, L. Gangloff, P. Legagneux, D. Dieumegard, G. Amaratunga, and W. Milne, Nature 437, 968 (2005).

${ }^{15}$ J. Bonard, J. Salvetat, T. Stöckli, L. Forró, and A. Châtelain, Appl. Phys. A 69, 245 (1999).

${ }^{16}$ R. Fowler and L. Nordheim, Proc. R. Soc. London, Ser. A 119, 173 (1928).

${ }^{17}$ X. Xu and G. R. Brandes, Appl. Phys. Lett. 74, 2549 (1999).

${ }^{18}$ J. Bonard, J. Salvetat, T. Stöckli, W. de Heer, L. Forró, and A. Châtelain, Appl. Phys. Lett. 73, 918 (1998).

${ }^{19}$ K. L. Jensen, J. Appl. Phys. 85, 2667 (1999).

${ }^{20}$ N. D. Lang, A. Yacoby, and Y. Imry, Phys. Rev. Lett. 63, 1499 (1989).

${ }^{21}$ Y. Gohda, Y. Nakamura, K. Watanabe, and S. Watanabe, Phys. Rev. Lett. 85, 1750 (2000).

${ }^{22}$ A. Maiti, J. Andzelm, N. Tanpipat, and P. von Allmen, Phys. Rev. Lett. 87, 155502 (2001).

${ }^{23}$ S. Han, M. H. Lee, and J. Ihm, Phys. Rev. B 65, 085405 (2002).

${ }^{24}$ K. Tada and K. Watanabe, Phys. Rev. Lett. 88, 127601 (2002).

${ }^{25}$ J. A. Driscoll and K. Varga, Phys. Rev. B 80, 245431 (2009).

${ }^{26}$ K. Varga, Z. Zhang, and S. Pantelides, Phys. Rev. Lett. 93, 176403 (2004).

${ }^{27}$ E. Runge and E. Gross, Phys. Rev. Lett. 52, 997 (1984).

${ }^{28}$ N. Troullier and J. L. Martins, Phys. Rev. B 43, 1993 (1991).

${ }^{29}$ U. von Barth and L. Hedin, J. Phys. C 5, 1629 (1972).

${ }^{30}$ J. P. Perdew and A. Zunger, Phys. Rev. B 23, 5048 (May 1981)

${ }^{31}$ K. Yabana and G. F. Bertsch, Phys. Rev. B 54, 4484 (1996).

${ }^{32}$ G. Halasz and A. Vibok, Int. J. Quantum Chem. 92, 168 (2003).

${ }^{33}$ D. Manolopoulos, J. Chem. Phys. 117, 9552 (2002).

${ }^{34}$ D. E. Manolopoulos, J. Chem. Phys. 117, 9552 (2002).

${ }^{35}$ P. Ehrenfest, Z. Phys. A: Hadrons Nucl. 45, 455 (1927).

${ }^{36}$ L. Verlet, Phys. Rev. 159, 98 (1967).

${ }^{37}$ D. T. Pierce, Phys. Scr. 38, 291 (1988).

${ }^{38}$ N. Müller, W. Eckstein, W. Heiland, and W. Zinn, Phys. Rev. Lett. 29, 1651 (1972).

${ }^{39}$ G. Baum, E. Kisker, A. Mahan, W. Raith, and B. Reihl, Appl. Phys. A 14, 149 (1977).

${ }^{40}$ D. T. Pierce and F. Meier, Phys. Rev. B 13, 5484 (1976)

${ }^{41}$ S. Hao, G. Zhou, J. Wu, W. Duan, and B.-L. Gu, Phys. Rev. B 69, 113403 (2004)

${ }^{42}$ S. Hao, G. Zhou, J. Wu, W. Duan, and B.-L. Gu, Chem. Phys. Lett. 401, 47 (2005)

${ }^{43}$ Y. Zhang, N. W. Franklin, R. J. Chen, and H. Dai, Chem. Phys. Lett. 331, 35 (2000), http://www.sciencedirect.com/science/article/pii/S000926 1400011623.

${ }^{44}$ S. Yuan, Y. Kong, F. Wen, and F. Li, Comput. Mater. Sci. 42, 83 (2008).

${ }^{45}$ G. Kresse and J. Hafner, Phys. Rev. B 47, 558 (1993). 